\title{
Adequação do Processo de Assistência Pré-natal entre as Usuárias do Sistema Único de Saúde em Juiz de Fora-MG
}

\author{
Adequacy of the Prenatal Care Process among Users of the Unified \\ Health Care System in Juiz de Fora-MG \\ Tadeu Coutinho ${ }^{1}$, Maria Teresa Bustamante Teixeira ${ }^{1}$, Sulamis Dain ${ }^{2}$, \\ Jane Dutra Sayd ${ }^{2}$, Larissa Milani Coutinho'
}

\begin{abstract}
RESUM0
Objetivos: avaliar a adequação do processo da assistência pré-natal oferecida às usuárias do SUS em Juiz de Fora/MG e comparar o atendimento nos principais serviços municipais. Métodos: estudo transversal desenvolvido com auditoria em 370 Cartões da Gestante selecionados por amostragem sistemática entre as pacientes a termo que utilizaram o SUS no atendimento ao parto, no primeiro semestre de 2002 e com pré-natal freqüentado em Juiz de Fora. Foi utilizado o teste do $\chi^{2}$ para comparar os serviços de procedência das pacientes (nível de significância: 5\%). A avaliação obedeceu a uma seqüência em três níveis complementares, sendo examinados: a utilização da assistência pré-natal (indice de Kessner: início e freqüência dos atendimentos) no nível 1; a utilização do pré-natal e dos exames laboratoriais básicos, segundo o Programa de Humanização no Pré-natal e Nascimento (tipagem $A B O / R h$, hemoglobina/hematócrito, VDRL, glicemia e exame de urina tipo 1), no nível 2; e a utilização de exames laboratoriais básicos e de procedimentos clínico-obstétricos obrigatórios numa consulta pré-natal (aferições de pressão arterial, peso, edema, altura uterina, idade gestacional, batimentos cardiofetais e apresentação fetal), no nível 3.

Resultados: a adequação do processo foi de apenas 26,7\% (nível 1), 1,9\% (nível 2) e 1,1\% (nível 3). Foram também observados cobertura pré-natal de 99,04\%, média de 6,4 consultas/ gestante, além da média de 17,4 semanas de idade gestacional na primeira consulta. Não houve diferenças significativas entre os diversos serviços municipais analisados.

Conclusões: o pré-natal das usuárias do SUS na cidade deve ser revisto qualitativamente, recomendando-se avaliações periódicas como instrumentos imprescindíveis de aperfeiçoamento. Aos gestores e profissionais de saúde cabem ações que aumentem a adesão às normas/rotinas do programa - principalmente a solicitação/registro dos exames complementares básicos -e propiciem melhor utilização do pré-natal pelas pacientes.
\end{abstract}

PALAVRAS-CHAVE: Assistência pré-natal. Gravidez normal. Pré-natal. Avaliação de assistência.

\footnotetext{
${ }^{1}$ Faculdade de Medicina da Universidade Federal de Juiz de Fora (UFJF)

${ }^{2}$ Instituto de Medicina Social da Universidade do Estado do Rio de Janeiro (IMS/UERJ).

Correspondência:

Tadeu Coutinho

Rua Batista de Oliveira, 1070/1502 - Bairro Granbery

36010-530 - Juiz de Fora - MG

Telefone: (32) 3211-9552/3215-3290

e-mail: coutinho@nextwave.com.br ou

coutinho@medicina.ufjf.br
}

\section{Introdução}

A assistência pré-natal tem merecido destaque crescente e especial na atenção à saúde materno-infantil, que permanece como um campo de intensa preocupação na história da Saúde Pública. No Brasil, a persistência de índices preocupantes de indicadores de saúde importan- 
tes, como os coeficientes de mortalidades materna e perinatal, tem motivado o surgimento de um leque de políticas públicas que focalizam o ciclo gravídico-puerperal. Entretanto, essas políticas têm se fundamentado principalmente no incremento da disponibilidade e do acesso ao atendimento pré-natal. A própria literatura especializada tem privilegiado a análise das características e dos resultados da assistência, relegando a um segundo plano o estudo da qualidade do conteúdo das consultas ${ }^{1,2}$. Esse conteúdo retrata com maior fidelidade o processo do atendimento e tem sido confirmado como preditor significativo do prognóstico do nascimento ${ }^{3}$.

É a avaliação do processo de um programa de saúde que analisa realmente o seu funcionamento, pois contempla os cuidados efetivamente fornecidos e recebidos na assistência. Assim, são igualmente importantes tanto as atividades dos profissionais envolvidos no diagnóstico e na terapêtica, quanto as atividade dos pacientes na procura e na utilização dos tratamentos disponibilizados ${ }^{4}$. Ademais, a desobediência às normas e rotinas do programa - principalmente a inadequação dos registros das consultas -, tem sido apontada pela própria literatura como um dos principais obstáculos para o aperfeiçoamento da qualidade da assistência pré-natal ${ }^{1}$.

O Programa de Humanização no Pré-Natal e Nascimento (PHPN/2000; Portaria/GM n ${ }^{\circ} 569$, de 1/6/2000) do Ministério da Saúde (MS) foi instituído com o objetivo declarado de assegurar a melhoria do acesso, da cobertura e da qualidade do acompanhamento pré-natal, da assistência ao parto e puerpério às gestantes e ao recém-nascido $^{5}$. Quanto à assistência à gestação, o PHPN/2000 determina os parâmetros de acesso e freqüência do atendimento e a solicitação de exames complementares básicos. No entanto, o Programa não menciona as atividades clínico-obstétricas de execução obrigatória numa consulta pré-natal, permitindo apenas uma análise parcial do processo da assistência à gestação.

O município mineiro de Juiz de Fora foi cadastrado no PHPN/2000 no ano de 2001. A cidade, apesar de estar próxima dos maiores centros urbanos brasileiros e alcançar usualmente boa avaliação nas pesquisas sobre qualidade de vida, não destoa do quadro nacional e tem persistentemente ostentado índices preocupantes das mortalidades materna e perinatal. São representativos os números de 2002, fornecidos pelos Comitês de Prevenção à Mortalidade Materna e Infantil na cidade: 89 óbitos maternos / 100 mil nascidos vivos (NV), além de mortalidade perinatal de 28,2 mortes/ $1000 \mathrm{NV}^{6}$.
Esse estudo se propôs a analisar a adequação do processo da assistência pré-natal prestada às usuárias do Sistema Único de Saúde (SUS) em Juiz de Fora, utilizando e complementando os critérios de avaliação preconizados pelo PHPN/2000. Além da avaliação do atendimento individualizado - objeto clínico, houve também preocupação com análise da dimensão coletiva do programa de cuidados pré-natais na cidade - conteúdo programático. Objetivou-se, também, a implantação de metodologia para avaliações periódicas do pré-natal.

\section{Pacientes e Métodos}

Trata-se de estudo de corte transversal, retrospectivo, baseado na auditoria de cartões da gestante.

Na seleção da amostra, foram obedecidos os seguintes critérios de inclusão: pacientes usuárias do SUS; portadoras do cartão da gestante a assistência pré-natal freqüentada em Juiz de Fora e que tiveram partos a termo realizados na Maternidade Therezinha de Jesus (MTJ), localizada nesta cidade, no período de $1^{\circ}$ de janeiro a 30 de junho de 2002 .

A MTJ é entidade beneficente, conveniada com a Faculdade de Medicina da Universidade Federal de Juiz de Fora (UFJF), e tem a quase totalidade da sua clientela (99,3\% em 2000) composta por pacientes usuárias do SUS. No período estudado, a MTJ foi uma das duas únicas instituições hospitalares que internaram as parturientes usuárias do SUS em Juiz de Fora, atendendo a $65,3 \%$ dos partos desse sistema na cidade, com aumento de $22,2 \%$ em relação ao primeiro semestre de 2001. A ausência de mecanismos efetivos de regionalização e hierarquização no atendimento ao ciclo gravídico-puerperal na cidade contribuiu para que a amostra pudesse ser considerada representativa da população-alvo.

A opção pelas gestações a termo (entre 37 semanas e 41 semanas e 6 dias) objetivou maior probabilidade de os cartões da gestante analisados espelharem de modo mais fidedigno e completo o processo da assistência pré-natal prestada na cidade. Além da procura espontânea, seja pela confirmação tardia ou pela maior aceitação da gravidez, a pressão familiar e a captação pelas unidades de saúde e ações comunitárias locais aumentam a adesão ao pré-natal com o evoluir da gestação.

Por meio do método de amostragem sistemática ${ }^{7}$, a população de estudo foi selecionada por 
sorteio e constituída por uma em cada quatro usuárias do SUS que tiveram seus partos registrados no livro de admissão da MTJ, no primeiro semestre de 2002. Foram selecionadas, inicialmente, 520 pacientes. A seguir, foram excluídas 66 parturientes $(12,7 \%)$ que freqüentaram a assistência pré-natal em outras cidades, 41 gestantes $(7,9 \%)$ que não apresentaram seus cartões de gestante durante a internação - incluindo 5 mulheres $(0,96 \%)$ que declararam não ter comparecido a consultas de pré-natal - e 43 pacientes $(8,3 \%)$ que tiveram partos pré-termo. A auditoria foi realizada, portanto, nos cartões das 370 gestantes que obedeceram a todos os critérios de seleção preestabelecidos.

Em relação às características sociodemográficas da amostra, a média de idade materna foi de 24,9 anos, havendo predomínio da faixa etária entre 20 e 29 anos (54,6 \%) e número significativo (20\%) de gestantes adolescentes (faixa de 1019 anos, segundo a OMS). Houve participação de $7,3 \%$ das pacientes com idade superior a 35 anos. As duas pacientes mais novas $(0,5 \%)$ tinham 14 anos e as três mães mais idosas $(0,8 \%)$ apresentavam 43 anos na época do parto. Houve predominância do registro da cor branca $(64,9 \%)$ e número significativo de pacientes solteiras $(45,4 \%)$, ao passo que apenas um terço $(33,3 \%)$ das pacientes tinha completado o ensino fundamental. Entre as adolescentes, $81,1 \%$ eram solteiras.

No tocante às suas características reprodutivas, $80,5 \%$ das pacientes relataram de uma a três gestações - com a participação das primigestas em $38,9 \%$ da amostra- e uma paciente $(0,3 \%)$ teve registradas 18 gravidezes.

Essas gestantes tinham sido atendidas no serviço tradicional do SUS ( $n=222 ; 60 \%$ ), pelo Programa de Saúde da Família (n=88; 23,8\%), no serviço da MTJ em convênio com a UFJF (n=21;5,7\%) e por convênios populares $(n=22 ; 5,9 \%)$ e clínicas particulares (n=14; 3,8\%). Não foi possivel definir a procedência de três pacientes. No caso de a gestante ter consultado em mais de um serviço, considerou-se como local de origem aquele em que foi realizado o maior número de atendimentos.

A análise dos dados coletados foi realizada por meio da sua categorização em três níveis complementares e de complexidade crescente (niveis 1,2 e 3), facilitando a identificação da origem dos problemas porventura existentes. Por meio desses três níveis, realizou-se tanto a avaliação da adequação do processo da assistência pré-natal em todo o município, quanto a comparação entre os serviços que atendem as gestantes em Juiz de Fora.

No nível 1, foi empregado o índice de Kessner ${ }^{8}$ para avaliar a utilização do pré-natal (iní- cio e número de consultas) segundo os critérios do PHPN $/ 2000^{5}$. No nível 2, ampliou-se a análise, com a adição dos exames complementares classificados como básicos pelo PHPN/2000: tipagem ABO-Rh, hemoglobina e hematócrito (Hb/Ht), glicemia em jejum, VDRL e urina tipo 1. No nível 3, a avaliação abrangeu a utilização do pré-natal, os exames laboratoriais básicos e os procedimentos clínico-obstétricos considerados essenciais para adequada assistência pré-natal: as aferições da idade gestacional, da altura uterina, da pressão arterial, do peso e do edema maternos, além dos batimentos cardíacos e apresentação fetais. Os pontos de corte relativos a esses procedimentos foram definidos pela possibilidade da sua aferição de acordo com a idade gestacional no momento da consulta ${ }^{2}$. Como podem ser aferidos em qualquer época da gravidez, a pressão arterial (PA), o peso, o edema, a altura uterina (AU) e o cálculo da idade gestacional (IG) devem ser registrados em todas as consultas, ao passo que a ausculta dos batimentos cardíacos fetais (BCF) e o diagnóstico da apresentação fetal podem ser detectados clinicamente e devem ser anotados a partir de 20 e 28 semanas de gestação, respectivamente.

O resultado final da avaliação permitiu classificar o processo da assistência pré-natal em adequado, inadequado ou intermediário. Considerouse como adequado o atendimento pré-natal que preencheu todos os seguintes critérios: início anterior a $14^{\mathrm{a}}$ semana de gravidez; seis ou mais consultas; número mínimo dos exames laboratoriais básicos (ABO-Rh e $\mathrm{Hb} / \mathrm{Ht}$ : um registro; glicemia, VDRL e urina tipo 1: dois registros) e dos procedimentos clínico-obstétricos obrigatórios (AU, IG, PA, edema e peso: cinco ou mais registros; $\mathrm{BCF}$ : quatro ou mais registros; apresentação fetal: dois ou mais registros). A inadequação foi definida pela ocorrência de pelo menos uma das seguintes condições: início após a $27^{\mathrm{a}}$ semana de gestação; duas ou menos consultas de pré-natal; nenhum registro de exame laboratorial; duas ou menos anotações de AU, IG, PA, edema, peso e BCF ou nenhum registro da apresentação fetal. As demais associações definiram o processo de atendimento como intermediário.

Os dados foram digitados em dupla entrada, tabulados e processados utilizando o programa EpiInfo (versão 6.01) ${ }^{9}$. Empregou-se o teste do $\chi^{2}$ na verificação comparativa entre os locais/serviços de procedência das gestantes, ao nível de significância de 5\%.

A pesquisa foi previamente avaliada e aprovada pela Comissão de Ética da Maternidade Therezinha de Jesus. 
Resultados

Foi observada grande variedade de modelos de cartão da gestante, inclusive dentro de um mesmo serviço. Os cartões preconizados pelo MS foram predominantes $(69,2 \%)$, mas apenas $29,4 \%$ corresponderam ao atual modelo oficial.

Foi identificada alta cobertura pré-natal (99,04\%) em Juiz de Fora. Entretanto, somente $29,7 \%$ das gestantes iniciaram o pré-natal no primeiro trimestre, ao passo que $9,2 \%$ procuraram assistência apenas nos últimos três meses de gestação. A idade gestacional média de ingresso foi de 17,4 semanas. A média de consultas por gestante foi de 6,4 , mas um terço $(33,8 \%)$ das pacientes não compareceu ao mínimo de 6 atendimentos, incluindo 5, 1\% que freqüentaram apenas uma ou duas consultas. Apesar de a data da última menstruação (DUM) não ter sido registrada corretamente em torno de um terço $(35,4 \%)$ dos cartões, a anotação da data provável do parto (DPP) foi adequada em $91,9 \%$ dos casos.

Houve, também, grande variabilidade no registro daqueles exames laboratoriais considerados como básicos pelo PHPN/ 20005 . No momento da internação, 7\% das gestantes não portavam a tipagem ABO-Rh. Não houve registros da dosagem de $\mathrm{Hb} / \mathrm{Ht}$ em 21,6\% dos cartões, do exame de uri-

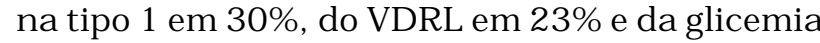
em 31,1\%. A testagem anti-HIV e o exame colpocitológico (Papanicolaou) também não foram anotados em 34,1\% e 91,4\% dos cartões analisados, respectivamente. Um total de 459 exames ultra-sonográficos foi registrado em 306 cartões $(82,7 \%$ das pacientes), mas em 25 anotações $(6,7 \%)$ não houve relato da idade gestacional na época do exame ecográfico inicial. Dentre os 281 exames ultra-sonográficos iniciais com relato da idade gestacional, houve predomínio da solicitação no segundo trimestre $(64,7 \%)$ e, ainda, porcentagem significativa no último trimestre (15,7\%). A média de realização de ultra-sonografia por gestante foi de 1,2, ao passo que o número máximo de exames realizados, em uma só paciente, foi de seis (Tabela 1).

Quanto aos procedimentos clínico-obstétricos listados na Tabela 2, a freqüência esperada (5 ou mais registros) das avaliações que independem de uma fase específica da gestação foi verificada na maioria dos cartões - idade gestacional (58, 1\%), pressão arterial $(77,8 \%)$, peso $(75,4 \%)$, e altura uterina $(72,7 \%)-$, com exceção para a anotação do edema $(48,1 \%)$. Com relação aos procedimentos dependentes da idade da gestação, as freqüências esperadas para a ausculta dos BCF (mínimo de 4 registros) e para o diagnóstico da apresentação fetal (duas ou mais anotações) foram verificadas em $79,5 \%$ e $35,7 \%$, respectivamente. Enquanto pelo menos um registro da pressão arterial esteve presente em todos os cartões, a avaliação da apresentação fetal foi o procedimento de menor freqüência, estando ausente, portanto, em $54,3 \%$ dos documentos estudados.

Tabela 1 - Exames complementares solicitados durante a assistência pré-natal das gestantes a termo atendidas na Maternidade Therezinha de Jesus de Juiz de Fora, no primeiro semestre de 2002.

\begin{tabular}{lcccc} 
Registros & Sim & Não & Um registro & Dois ou mais \\
& $\mathbf{n ~ ( \% )}$ & $\mathbf{n ~ ( \% )}$ & $\mathbf{n}(\%)$ & $\mathbf{n ~ ( \% ) ~}$ \\
\hline Tipo (ABO)-Rh & 344 & 26 & - & - \\
& $(93,0 \%)$ & $(7,0 \%)$ & & \\
Hemoglobina/ & 290 & 80 & 235 & 55 \\
hematócrito & $(78,4)$ & $(21,6 \%)$ & $(63,5)$ & $(14,9)$ \\
Urinálise & 259 & 111 & 208 & 51 \\
(urina tipo 1) & $(70,0)$ & $(30,00)$ & $(56,2)$ & $(13,8)$ \\
VDRL & 284 & 86 & 243 & 41 \\
& $(76,8)$ & $(23,2)$ & $(65,7)$ & $(11,1)$ \\
Glicemia & 255 & 115 & 194 & 61 \\
& $(68,9)$ & $(31,1)$ & $(52,4)$ & $(16,5)$ \\
Teste anti-HIV & 244 & 126 & 230 & 14 \\
& $(65,9)$ & $(34,1)$ & $(62,1)$ & $(3,8)$ \\
Ultra-sonografia & 306 & 64 & - & - \\
& $(82,7)$ & $(17,3)$ & & \\
\hline
\end{tabular}

Tabela 2 - Procedimentos clínico-obstétricos realizados na assistência pré-natal das gestantes a termo atendidas na Maternidade Therezinha de Jesus de Juiz de Fora, no primeiro semestre de 2002.

\begin{tabular}{|c|c|c|c|c|}
\hline Registros & $\begin{array}{l}\text { Sim } \\
n(\%)\end{array}$ & $\begin{array}{l}\text { Não } \\
\text { n (\%) }\end{array}$ & $\begin{array}{c}\text { Um a quatro } \\
n(\%)\end{array}$ & $\begin{array}{c}\text { Cinco ou mais } \\
\mathrm{n}(\%)\end{array}$ \\
\hline $\begin{array}{l}\text { Altura uterina } \\
(\mathrm{AU})\end{array}$ & $\begin{array}{c}368 \\
(99,5)\end{array}$ & $\begin{array}{c}02 \\
(0,5)\end{array}$ & $\begin{array}{c}99 \\
(26,8)\end{array}$ & $\begin{array}{c}269 \\
(72,7)\end{array}$ \\
\hline $\begin{array}{l}\text { Idade gestacional } \\
\text { (IG) }\end{array}$ & $\begin{array}{c}303 \\
(81,9)\end{array}$ & $\begin{array}{c}67 \\
(18,1)\end{array}$ & $\begin{array}{c}88 \\
(23,8)\end{array}$ & $\begin{array}{c}215 \\
(58,1)\end{array}$ \\
\hline $\begin{array}{l}\text { Pressão arterial } \\
\text { (PA) }\end{array}$ & $\begin{array}{c}370 \\
(100)\end{array}$ & $\begin{array}{c}0,0 \\
(0,0)\end{array}$ & $\begin{array}{c}82 \\
(22,2)\end{array}$ & $\begin{array}{c}288 \\
(77,8)\end{array}$ \\
\hline $\begin{array}{l}\text { Peso materno } \\
\text { (PM) }\end{array}$ & $\begin{array}{c}369 \\
(99,7)\end{array}$ & $\begin{array}{c}01 \\
(0,3)\end{array}$ & $\begin{array}{c}90 \\
(24,3)\end{array}$ & $\begin{array}{c}279 \\
(75,4)\end{array}$ \\
\hline Edema & $\begin{array}{c}265 \\
(71,6)\end{array}$ & $\begin{array}{c}105 \\
(28,4)\end{array}$ & $\begin{array}{c}87 \\
(23,5)\end{array}$ & $\begin{array}{c}178 \\
(48,1)\end{array}$ \\
\hline $\begin{array}{l}\text { Batimentos } \\
\text { cardíacos fetais } \\
(\mathrm{BCF})^{1}\end{array}$ & $\begin{array}{c}368 \\
(99,5)\end{array}$ & $\begin{array}{c}02 \\
(0,5)\end{array}$ & $\begin{array}{c}74 \\
(20,0)\end{array}$ & $\begin{array}{c}294 \\
(79,5)\end{array}$ \\
\hline $\begin{array}{l}\text { Apresentação } \\
\text { fetal }^{2}\end{array}$ & $\begin{array}{c}169 \\
(45,7)\end{array}$ & $\begin{array}{c}201 \\
(54,3)\end{array}$ & $\begin{array}{c}37 \\
(10,0)\end{array}$ & $\begin{array}{c}132 \\
(35,7)\end{array}$ \\
\hline $\begin{array}{l}\text { Vacinação } \\
\text { antitetânica }\end{array}$ & $\begin{array}{c}157 \\
(42,4)\end{array}$ & $\begin{array}{c}213 \\
(57,6)\end{array}$ & - & - \\
\hline Prescrição & $\begin{array}{c}257 \\
(69,5)\end{array}$ & $\begin{array}{c}113 \\
(30,5)\end{array}$ & - & - \\
\hline
\end{tabular}

${ }^{1}$ Um a três registros/quatro ou mais registros.

${ }^{2} U \mathrm{~m}$ registro/dois ou mais registros. 
A vacinação antitetânica foi registrada em $42,4 \%$ dos casos e o relato de cobertura vacinal anterior à gestação foi encontrado em apenas $26 \%$ dos cartões. Foi registrada a prescrição de pelo menos um medicamento em $69,5 \%$ dos cartões. Utilizado para a prevenção e tratamento de anemia, o ferro, principalmente na forma de sulfato ferroso, teve o maior número de registros (252 cartões). Apesar de estarem presentes em 322 cartões $(87 \%)$, as curvas altura uterina $\mathrm{x}$ idade gestacional (AU $x$ IG) e peso materno $x$ idade gestacional (PM x IG) tiveram seu preenchimento completo verificado em apenas $12,1 \%$ e $6,5 \%$ desses cartões, respectivamente.

Como pode ser observado na Tabela 3 , o estudo seqüencial e complementar dos registros da utilização da assistência, dos exames laboratoriais básicos e da freqüência dos procedimentos clínico-obstétricos obrigatórios nas consultas permitiu constatar os baixos niveis de adequação do processo do pré-natal prestado às usuárias do SUS no município de Juiz de Fora: $27,6 \%$ no nível 1; 1,9\% no nível 2; e 1,1\% no nível 3. Utilizando os mesmos critérios, a avaliação comparativa entre os locais/serviços de procedência das gestantes estudadas não revelou diferenças estatisticamente significativas $(\mathrm{p}>0,05)$.

Tabela 3 - Adequação do processo de assistência pré-natal prestada no município de Juiz de Fora às gestantes a termo atendidas, pelo SUS, na Maternidade Therezinha de Jesus de Juiz de Fora, no primeiro semestre de 2002.

\begin{tabular}{lccc}
\hline $\begin{array}{l}\text { Avaliação do } \\
\text { Processo }\end{array}$ & $\begin{array}{c}\text { Adequado } \\
\mathbf{n}(\%)\end{array}$ & $\begin{array}{c}\text { Inadequado } \\
\mathbf{n}(\%)\end{array}$ & $\begin{array}{c}\text { Intermediário } \\
\mathbf{n}(\%)\end{array}$ \\
\hline Nível 1 & $102(27,6)$ & $44(11,9)$ & $224(60,5)$ \\
Nível 2 & $7(1,9)$ & $179(48,4)$ & $184(49,7)$ \\
Nível 3 & $4(1,1)$ & $179(48,4)$ & $187(50,5)$ \\
\hline
\end{tabular}

Nível 1 - Utilização do pré-natal; Nível 2: utilização do pré-natal + exames laboratoriais básicos; Nível 3: utilização do pré-natal + exames + procedimentos clínico-obstétricos.

\section{Discussão}

$\mathrm{Na}$ metodologia adotada, foi empregada a auditoria, uma das técnicas de obtenção de dados para avaliação da qualidade da atenção médica. A auditoria médica pode ser realizada simultânea ou retrospectivamente ao processo de investigação e, ao utilizá-la, o pesquisador com experiência na área pode avaliar tanto o processo como o produto. A importância dos registros médicos na prática clínica, ao influenciar os processos de cuidados, faz com que a auditoria possa refletir a qualidade da atenção prestada ${ }^{10}$. Dessa forma, pressupõe-se que, se não foi registrado, o procedimento médico não deve ter sido realizado.
Para estudar a utilização do atendimento pelas gestantes foi adotado o índice de Kessner, um dos métodos mais difundidos para a análise quantitativa da assistência pré-natal, que, mesmo tendo sofrido várias modificações desde a sua descrição em 1972, manteve intacta a sua estrutura nuclear: avaliação do início e da freqüência do atendimento ${ }^{8}$.

A análise de adequação foi utilizada por ser mais simples e menos dispendiosa que as outras modalidades - análises de plausibilidade ou probabilidade -, poder usar dados secundários e requerer, em geral, uma única medida a ser comparada com critérios preestabelecidos, prescindindo de grupos controle. Se a eficácia da intervenção analisada já foi comprovada, como é o caso da assistência pré-natal, uma única avaliação é suficiente para responder à maioria das perguntas formuladas pelos gestores nos níveis municipal, estadual e nacional ${ }^{11}$. Entretanto, naquelas avaliações de um mesmo programa em diferentes períodos de tempo, há necessidade de duas medidas, no mínimo, para detectar tendências porventura existentes. A avaliação de plausibilidade exige um grupo controle do tipo histórico, interno ou externo, sendo utilizada para descartar os fatores externos - também denominados fatores de confundimento - que podem ser responsáveis pelos efeitos observados num determinado estudo. A análise de probabilidade é mais complexa, representa o padrão-ouro das pesquisas acadêmicas de eficácia e requer randomização das unidades do estudo, sendo importante para o desenvolvimento de novas intervenções.

A multiplicidade de modelos dos cartões utilizados, incluindo aqueles empregados pelos serviços pertencentes ao SUS, sabidamente dificulta o estabelecimento de uma rotina de preenchimento, a análise comparativa de algumas variáveis e a implantação de um sistema adequado de referência/contra-referência.

A análise das características socioeconômicas e demográficas da amostra estudada revelou porcentagens significativas de adolescentes (20\%), de solteiras $(45,4 \%)$, de multigestas $(61,1 \%)$ e de pacientes com ensino fundamental incompleto $(60,8 \%)$. Segundo a literatura, todas essas condições se relacionam, no seu conjunto ou isoladamente, com baixa utilização da assistência prénatal, contribuindo para o aumento da morbimortalidade materno-fetal ${ }^{12-19}$. Neste estudo, por exemplo, em comparação com as gestantes analfabetas $(0,8 \%)$, as portadoras de escolaridade superior a oito anos apresentaram percentuais maiores tanto da procura pelo prénatal no primeiro trimestre $(33,0 \%$ vs. $0,0 \%)$, quan- 
to da média de consultas $(6,9$ vs. 4,3). A constatação da predominância do registro da cor branca $(64,9 \%)$ reflete as mesmas dificuldades encontradas pelo Instituto Brasileiro de Geografia e Estatística (IBGE) no Censo 2000 (não consta referência), que apontou o percentual irreal de $6,2 \%$ (cerca de 10 milhões) de população de cor preta, longe de refletir a realidade brasileira. Em termos socioeconômico-culturais, a sobreposição das questões de gênero e de cor alavanca as desigualdades, tornando mais relevante esse dado. Ademais, sob o ponto de vista obstétrico, há maior ocorrência de hipertensão arterial, anemia falciforme e vícios pélvicos nas mulheres melanodérmicas ${ }^{13,18,20}$.

A cobertura da assistência pré-natal encontrada (99,04\%) foi compativel com os dados fornecidos pelo SINASC/JF 2001 (99,0\%) (não consta referência) e superior àquelas registradas na literatura nacional ${ }^{2,21,22}$. A cobertura de um programa constitui indicador importante da sua operacionalidade ${ }^{23}$. Entretanto, essa alta cobertura foi acompanhada de baixa utilização do prénatal (Tabela 3). A maior procura pelos cuidados pré-natais no segundo trimestre $(61,1 \%)$ retrata o baixo nível de sensibilização e conscientização da comunidade acerca da relevância do seu início precoce. O total de consultas de pré-natal preconizado pela OMS não deve ser inferior a seis, e qualquer número abaixo desta cifra já é considerado como atendimento deficitário ${ }^{10}$. Neste estudo, parcela considerável $(33,8 \%)$ das gestantes não compareceu a esse número mínimo de consultas.

Em relação aos exames laboratoriais, são preocupantes os altos percentuais de falta de registro de variáveis relacionadas a intercorrências maternas importantes, como a anemia, as infecções do trato urinário, o diabete melito e a sífilis. A ausência de registro do teste anti-HIV em um terço dos cartões, com a sua positividade em três pacientes - correspondendo a 1,2\% dos exames realizados e a $0,8 \%$ da amostra -, aponta a necessidade de esforço maior das equipes de saúde de Juiz de Fora na orientação sobre a prevenção da transmissão materno-fetal da infecção.

Em contraposição, os registros de realização da ultra-sonografia $(82,7 \%)$ foram muito superiores aos números relatados na literatura brasileira, com exceção de Caxias do Sul - RS $(96,8 \%)^{10,22,24}$. O exame ultra-sonográfico tornouse componente importante da assistência prénatal moderna e uma das suas principais indicações consiste na correção e/ou determinação da idade gestacional, como pôde ser demonstrado, neste estudo, pela diferença encontrada entre os registros corretos da DUM e da DPP (64,6\% vs.
91,9\%). Entretanto, houve clara priorização da ultra-sonografia obstétrica em detrimento dos exames laboratoriais básicos. O registro inadequado do exame colpocitológico $(8,6 \%)$ torna-se ainda mais significativo por ser a gestação o momento ideal - e, às vezes, único - para implementar ou consolidar as orientações e as práticas preventivas para a saúde feminina.

Como conseqüência, a adição dos exames laboratoriais básicos à utilização do pré-natal (nível 2), ao proporcionar avaliação mais qualitativa, resultou na redução mais acentuada da adequação do pré-natal. Aqui, as medidas corretivas devem ser mais abrangentes, pois a ausência do registro de um determinado exame pode se dever a: falta de solicitação do exame pela equipe técnica; falta de registro adequado pelos profissionais, apesar do exame ter sido solicitado e realizado; dificuldades para o agendamento e a realização do exame pelo SUS; realização do exame, porém sem retorno ao serviço de saúde; ou não-realização do exame motivada pela própria paciente.

A análise do acréscimo dos procedimentos clínico-obstétricos (Nivel 3) evidenciou, também, altos percentuais de falta de registro. A responsabilidade pela realização ou a anotação desses procedimentos cabe fundamentalmente à equipe técnica e, neste estudo, o atendimento majoritário $(60 \%)$ foi realizado por tocoginecologistas, como integrantes das equipes tradicionais do SUS. A ausência da variável edema em alguns tipos de cartão pode explicar o seu registro inadequado. $\mathrm{O}$ edema tem sido desvalorizado como fator complicador da gestação - presença em 50 a 80\% das gestações normais ${ }^{13}$ e ausência em cerca de um quarto daquelas complicadas com eclâmpsia ${ }^{25}$ -, ocasionando a omissão desse sinal clínico na confecção de algumas modalidades de cartão da gestante. Porém, a ausência de anotação da idade gestacional não pode ser explicada apenas pelos altos índices de desconhecimento das datas da última menstruação (35,4\%). Uma anamnese mais acurada pode contribuir para a diminuição desses índices e do número de exames solicitados apenas para a definição da idade gestacional, melhorando a relação custo-benefício da assistência pré-natal.

Quanto à falta de registro da apresentação fetal em mais da metade dos cartões, podem ser aventadas algumas hipóteses: o despreparo técnico para a realização de todas as etapas do exame obstétrico clássico, a pouca valorização desse procedimento e/ou a comodidade da sua substituição pela avaliação ultra-sonográfica. O diagnóstico da apresentação fetal realizada no último trimestre da gestação deve ser essencialmente clí- 
nico, sendo importante na decisão sobre o tipo de parto e na previsão de possíveis intercorrências durante o trabalho de parto.

Em mais da metade dos cartões $(57,6 \%)$ não houve registro de vacinação antitetânica, caracterizando cobertura inferior àquelas relatadas na literatura brasileira ${ }^{10,22,24}$. A baixa freqüência de anotação da imunização das gestantes denota clara incompreensão acerca da importância desse registro e/ou da própria prevenção do tétano neonatal. A ausência das curvas que relacionam o crescimento uterino e o peso materno com a idade gestacional (13\% dos cartões) também não pode justificar os índices extremamente baixos de preenchimento completo das curvas disponiveis (12,1 e 6,5\%, respectivamente).

Como conclusão, o processo da assistência pré-natal prestada às usuárias do SUS em Juiz de Fora, a despeito da alta cobertura, apresentou baixa adequação em todos os locais/serviços e níveis estudados. Por terem sido analisados apenas critérios considerados mínimos para um atendimento de baixa complexidade, esses resultados se mostram ainda mais significativos. É evidente a necessidade de uma discussão abrangente - envolvendo obrigatoriamente gestores, profissionais de saúde e comunidade - sobre a implantação de ações corretivas imediatas e de avaliações periódicas do pré-natal no município. Nesse contexto, a participação efetiva dos tocoginecologistas é indispensável e a sua atuação deve servir como parâmetro para um atendimento pré-natal adequado.

Todas essas medidas se revestem de uma maior importância numa sociedade caracterizada por elevada desigualdade social, que é refletida, no campo da saúde, pela existência de relação inversa entre a disponibilidade da assistência e as necessidades da população assistida. No entanto, são encorajadoras as experiências de outros locais (cidades, regiões ou países) que, com nível de desenvolvimento igual ou inferior ao nosso, conseguiram reduzir a mortalidade materno-infantil por meio de sistemas de saúde organizados e abrangentes e que, prioritariamente, utilizaram tecnologias simplificadas e viáveis sob o ponto de vista econômico.

\section{ABSTRACT}

Purpose: to evaluate the adequacy of the prenatal care process offered to users of the Unified Health Care System (SUS) in Juiz de Fora-MG and to compare the municipal services.
Methods: a transversal study with auditing was carried out on the records of 370 pregnant women, selected by systematic sampling among women who carried their pregnancies to term and gave birth using SUS services in the first semester of 2002, with prenatal care given in Juiz de Fora. For statistical analysis the $\chi^{2}$ test was used to compare the municipal services (level of significance: $5 \%$ ). The evaluation followed a threetiered complementary sequence, using: the utilization of prenatal care (Kessner index: beginning and frequency of care) at level 1; the utilization of prenatal care and basic laboratory tests, according to the Humanization Program of Prenatal Care and Birth (ABO-Rh, hemoglobin/hematocrit, VDRL, glycemia and urinalysis), at level 2; and the utilization of prenatal care, the basic laboratory tests and the obligatory clinical-obstetric procedures during a prenatal visit (assessment of blood pressure, weight, edema, uterine fundal height, gestational age, fetal heart rate and fetal presentation), at level 3.

Results: the observed adequacy of the process was only $26.7 \%$ (level 1), 1.9\% (level 2) and 1.1\% (level 3). We also observed a prenatal coverage of $99.04 \%$, an average of 6.4 visits per pregnant woman, and an average gestational age of 17.4 weeks at the time of the first prenatal visit. There were no significant differences between the municipal services.

Conclusions:prenatal care offered to SUS users in Juiz de Fora should be reviewed from a qualitative standpoint, and periodic evaluations as necessary instruments of improvement are recommendable. Managers and professionals should undertake actions aimed at increasing compliance with norms/routines of the program - principally the request/recording of basic complementary tests - and which guarantee increased utilization of prenatal care.

KEYWORDS: Prenatal care. Normal pregnancy. Care audit.

\section{Referências}

1. Peoples-Sheps MD, Kalsbeek WD, Siegel E, Dewees C, Rogers M, Schwartz R. Prenatal records: a national survey of content. Am J Obstet Gynecol $1991 ; 164: 514-21$.

2. Silveira DS, Santos IS, Costa JSD. Atenção pré-natal básica: uma avaliação da estrutura e do processo. Cad Saúde Pública 2001; 17:131-9.

3. Kogan MD, Alexander GR, Kotelchuck M, Nagey DA, Jack BW. Comparing mother's reports on the content of prenatal care received with recommended national guidelines for care. Public Health Rep 1994; 109:637-46.

4. Donabedian A. The quality of care. How can it be assessed? JAMA 1988; 260:1743-8.

5. Ministério da Saúde. Secretaria de Políticas de Saúde. Programa de Humanização no Pré-natal e Nascimento (PHPN). Brasília; 2000. 
6. Boletim de Epidemiologia. Juiz de Fora: Prefeitura/ Departamento de Epidemiologia; $4^{\circ}$ trim 2002.

7. Torres TZG. Epidemiologia. $1^{\mathrm{a}}$ ed. São Paulo: Atheneu; 2002. Amostragem; p.283-94.

8. Alexander GR, Kotelchuck M. Quantifying the adequacy of prenatal care: a comparison of indices. Public Health Rep 1996; 111:408-18.

9. Dean AG, Dean JA, Coulombier D, et al. Epi Info: computer programs for epidemiology. Version 6.01. Atlanta: Centers for Disease Control and Prevention; 1994.

10. Dias-da-Costa JS, Madeira ACC, Luz RM, Britto MAP. Auditoria médica: programa de pré-natal em posto de saúde na região Sul do Brasil. Rev Saúde Pública 2000; 34:329-36.

11.Habicht JP, Victora CG, Vaughan JP. Evaluation designs for adequacy, plausibility and probability of public health programme performance and impact. Int J Epidemiol 1999; 28:10-8.

12.Federação Brasileira das Sociedades de Ginecologia e Obstetrícia. Assistência pré-natal: manual de orientação. Rio de Janeiro; 2000.

13. Cunningham FG, Gant NF, Leveno KJ, Gilstrap III LC, Hauth JC, Wenstrom KD. Prenatal care. In: Williams Obstetrics. $21^{\text {st }}$ ed. New York: McGrawHill; 2001. p.221-47.

14.Lambrou NC, Morse AN, Wallach EE. The Johns Hopkins manual of gynecology and obstetrics. $1^{\text {st }}$ ed. Baltimore: Lippincott Williams \& Wilkins; 1999. p.19-39.

15. Costa AAR, Ribas MSSS, Amorim MMR, Santos LC. Mortalidade materna na cidade do Recife. Rev Bras Ginecol Obstet 2002; 24: 455-62.
16. Monteiro CA, França Júnior I, Conde WL. Evolução da assistência materno-infantil na cidade de São Paulo (1984-1996). Rev Saúde Pública 2000; 34 Supl:19-25.

17. Brasil: pesquisa nacional sobre demografia e saúde1996. Rio de Janeiro: BEMFAM/Macro International; 1997.

18. Rezende J, Montenegro CAB. Obstetrícia fundamental. 9a ed. Rio de Janeiro: Guanabara Koogan; 2003.

19.Delgado-Rodríguez M, Gómez-Olmedo M, BuenoCavanillas A, Gálvez-Vargas R. Unplanned pregnancy as a major determinant in inadequate use of prenatal care. Prev Med 1997; 26:834-8.

20. Coutinho T. Alterações hematológicas na gravidez. In: SOGIMIG. Ginecologia \& Obstetrícia. $3^{\mathrm{a}}$ ed. Rio de Janeiro: Medsi; 2003. p.692-700.

21. Halpern R, Barros FC, Victora CG, Tomasi E. Atenção pré-natal em Pelotas, Rio Grande do Sul, Brasil, 1993. Cad Saúde Pública 1998; 14:487-92.

22.Trevisan MR, De Lorenzi DRS, Araújo NM, Ésber K. Perfil da assistência pré-natal entre usuárias do Sistema Único de Saúde em Caxias do Sul. Rev Bras Ginecol Obstet 2002; 24: 293-9.

23. Sala A, Nemes MIB, Cohen DD. Metodologia de avaliação do trabalho na atenção primária à saúde. Cad Saúde Pública 1998; 14:741-51.

24.Santos IS, Baroni RC, Minotto I, Klumb AG. Critérios de escolha de postos de saúde para acompanhamento pré-natal em Pelotas, RS. Rev Saúde Pública 2000; 34:603-9.

25. Mattar F, Sibai BM. Eclampsia. VIII. Risk factors for maternal morbidity. Am J Obstet Gynecol 2000; 182:307-12.

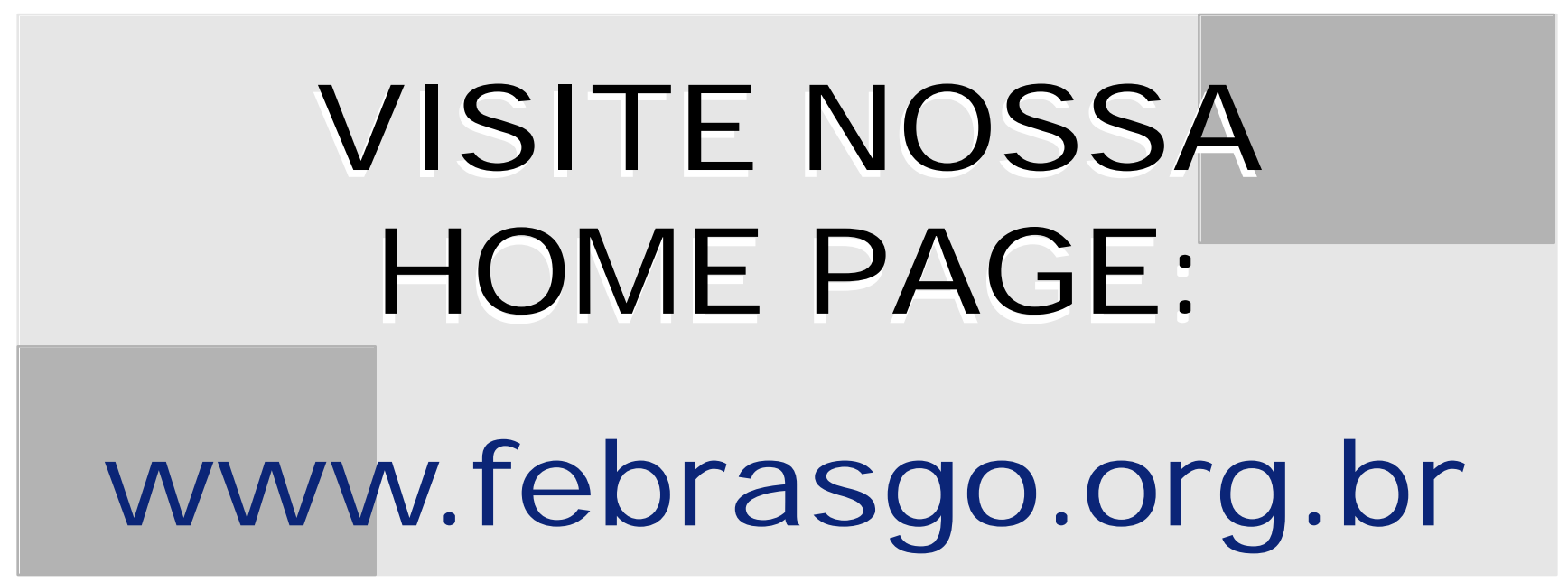

